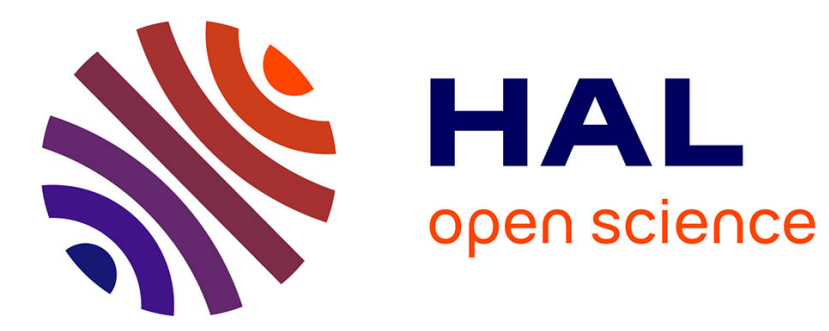

\title{
Effect of sliding filters on the soliton optical phase jitter in constant-dispersion systems
}

\author{
M. Hanna, D. Boivin, P.A. Lacourt, Jean-Pierre Goedgebuer
}

\section{To cite this version:}

M. Hanna, D. Boivin, P.A. Lacourt, Jean-Pierre Goedgebuer. Effect of sliding filters on the soliton optical phase jitter in constant-dispersion systems. Optics Communications, 2004, 231 (1-6), pp.181185. 10.1016/j.optcom.2003.11.066 . hal-00096915

\section{HAL Id: hal-00096915 https://hal.science/hal-00096915}

Submitted on 15 Apr 2021

HAL is a multi-disciplinary open access archive for the deposit and dissemination of scientific research documents, whether they are published or not. The documents may come from teaching and research institutions in France or abroad, or from public or private research centers.
L'archive ouverte pluridisciplinaire HAL, est destinée au dépôt et à la diffusion de documents scientifiques de niveau recherche, publiés ou non, émanant des établissements d'enseignement et de recherche français ou étrangers, des laboratoires publics ou privés. 


\title{
Effect of sliding filters on the soliton optical phase jitter in constant-dispersion systems
}

\author{
Marc Hanna ${ }^{\mathrm{a}, *}$, David Boivin ${ }^{\mathrm{a}}$, Pierre-Ambroise Lacourt ${ }^{\mathrm{a}}$, \\ Jean-Pierre Goedgebuer ${ }^{b}$

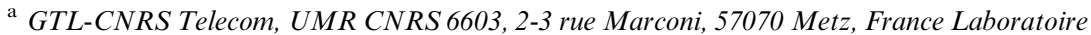 \\ b'optique P.M. Duffieux, UMR CNRS 6603, Route de Gray, 25030 Besançon Cedex, France
}

\begin{abstract}
The influence of sliding filters on the phase jitter of soliton pulses propagating in single-channel constant-dispersion systems is studied using the perturbation theory. Although sliding filters allow the reduction of the amplified radiative background, they introduce coupling between the soliton amplitude and frequency, and between its timing and phase, which result in a growth of the phase jitter as the cube of the propagation distance. Comparisons of the analytical results with Monte Carlo simulations validate the perturbation theory.
\end{abstract}

Keywords: Solitons; Optical communications; Optical phase jitter; Differential phase-shift keying

The use of differential phase shift keying (DPSK) has recently allowed the demonstration of impressive transmission capacities in the context of long-haul fiber-optic communication systems [15]. In these systems, the error-free distance is mainly limited by random fluctuations of the phase caused by the amplified spontaneous emission (ASE) that occurs in optical amplifiers. Without control, amplitude-to-phase noise conversion occurs through the self-phase modulation (SPM) [6] and cross-phase modulation (XPM) [7]

\footnotetext{
${ }^{*}$ Corresponding author. Tel.: +333-8720-3926; fax: +3338720-3940.

E-mail address: mhanna@georgiatech-metz.fr (M. Hanna).
}

effects, producing a phase variance that grows as the cube of the propagation distance. In-line filtering was shown to reduce phase jitter efficiently $[8,6]$ through the damping of amplitude noise, which result in a linear increase of the phase jitter with distance. The main drawback of this technique is that the ASE noise that has exactly the soliton center frequency is less attenuated than the soliton itself and grows exponentially with distance, creating a strong continuum wave that deteriorates the transmission.

To overcome this limitation, the idea of slidingfrequency guiding filters was proposed [9] and demonstrated [10-12] in the context of timing jitter. It allows the creation of an optical line that is opaque to noise but transparent for solitons. In 
this Letter, we present for the first time to our knowledge an analysis of the effect of sliding filters on the optical phase jitter of soliton pulses in single-channel constant-dispersion links. An analytical expression for the phase standard deviation is derived by use of the soliton perturbation theory. This result is validated by comparison with Monte Carlo simulations. We show that, as in the case of timing jitter, sliding filtering allows an efficient reduction of the phase jitter created by the interaction with the continuum, thus allowing the use of narrower optical filters. However, the coupling between amplitude and frequency created by the sliding makes the phase jitter increase as the propagation distance cube. This evolution is different from the timing jitter behavior, which only increases linearly in the presence of sliding filters. The choice of the optimal sliding rate involves a trade-off between the reduction of the continuum and the increase of the phase jitter.

The propagation of optical pulses in a slidingfiltered constant-dispersion amplified link is described by the perturbed nonlinear Schrödinger equation

$\mathrm{i} \partial_{z} u+\frac{1}{2} \partial_{t t}^{2} u+|u|^{2} u=\epsilon P(u)$,

where $P$ represents the perturbation and includes both the sliding filters and ASE noise, $\epsilon$ is a small parameter, and a standard normalization of the NLSE has been used. Properly chosen transformation and uniformly accelerated frame [13] allow us to write the perturbation due to sliding-frequency filters as $\epsilon P=\mathrm{i}\left[\delta u+(3 / 4) k_{\mathrm{f}} \partial_{t t}^{2} u\right]-\omega_{\mathrm{f}}^{\prime} t u$, where $\delta$ is the excess gain required to overcome the loss imposed on the solitons by the filters, $k_{\mathrm{f}}$ is the filter strength, and $\omega_{\mathrm{f}}^{\prime}$ is the sliding rate. Unless otherwise indicated, notations of [13] are used throughout the derivation. The soliton solution of the non-perturbed NLSE is given by

$u_{\mathrm{s}}(t)=A \operatorname{sech}[A(t-\alpha)] \exp (\mathrm{i} \phi-\mathrm{i} \omega t)$,

where $A$ is the amplitude, $\alpha$ the temporal position, $\omega$ the angular frequency, and $\phi$ the phase of the soliton. Each amplifier imparts a zero-mean random shift to the soliton parameters, with variances given by [13]

$\left\langle\delta A^{2}\right\rangle=A \epsilon_{n} n_{\mathrm{sp}} F(G)$, $\left\langle\delta \omega^{2}\right\rangle=\frac{A}{3} \epsilon_{n} n_{\mathrm{sp}} F(G)$,

$\left\langle\delta \alpha^{2}\right\rangle=\frac{\pi^{2}}{12 A} \epsilon_{n} n_{\mathrm{sp}} F(G)$,

$\left\langle\delta \phi^{2}\right\rangle=\frac{1}{3 A}\left(\frac{\pi^{2}}{12}+1\right) \epsilon_{n} n_{\mathrm{sp}} F(G)$,

where $\epsilon_{n}$ is the ratio of the photon energy to the unit energy (in soliton units), $n_{\mathrm{sp}}$ is the spontaneous emission factor, $G$ is the gain of the amplifier and $F(G)=(G-1)^{2} /[G \ln (G)]$ is the ratio of the soliton peak power at the amplifier output to the peak power of the average soliton. The perturbation theory can be used to calculate the evolution of the soliton parameters as a function of distance [13]. The filters guide the amplitude and frequency to a fixed point given by $A=1$ and $\omega=-\omega_{\mathrm{f}}^{\prime} / k_{\mathrm{f}}=\Delta$. The evolution of small variations around this fixed point $A=1+a$ and $\omega=\Delta+d$ are given by

$\left[\begin{array}{l}a(z) \\ d(z)\end{array}\right]=\left[\begin{array}{cc}t_{1}+t_{2} & \sqrt{3 / 2}\left(t_{1}-t_{2}\right) \\ \sqrt{2 / 3}\left(t_{1}-t_{2}\right) & t_{1}+t_{2}\end{array}\right]\left[\begin{array}{l}a_{0} \\ d_{0}\end{array}\right]$,

where $\gamma_{i}=k_{\mathrm{f}}(1 \pm|\Delta| \sqrt{6}), t_{i}(z)=\exp \left(-\gamma_{i} z\right)$, and $a_{0}$ and $d_{0}$ are initial values of the amplitude and frequency. The magnitude of the parameter $\Delta$ must be less than $1 / \sqrt{6}$ to ensure stable propagation of the pulses. For a given filter strength, this condition fixes an upper limit for the sliding rate.

To calculate the phase jitter, we change the referential and shift the fixed point frequency to zero. The evolution of the temporal position and phase are given by

$\alpha_{z}^{\prime}=-d$,

$\phi_{z}^{\prime}=a+\omega_{f}^{\prime} \alpha$.

All parameters have an influence on the evolution of the phase. Since the amplitude and frequency are coupled by the sliding filters, they both intervene in the first term on the right-hand side of Eq. (9). The temporal position modifies the phase jitter through the second term of this equation. At a given distance $z$, we can calculate the contribution of each amplifier to the phase by using the 
initial values given by Eqs. (3)-(6) and the parameter evolution Eqs. (7)-(9). Adding up all these contributions, taking the variance, approximating the discrete sum over the amplifiers by a continuous integral, and for distances such that $\gamma_{2} z \gg 1$, we obtain the following expression for the phase variance:

$\left\langle\delta \phi^{2}(z)\right\rangle=\frac{\epsilon_{n} n_{\mathrm{sp}} F(G)}{3 z_{A}}\left[A_{1} z+A_{2} z^{2}+A_{3} z^{3}\right]$,

with

$A_{1}=3 a_{1}^{2}+3 a_{2}^{2}+6 a_{1} a_{2}+b_{1}^{2}+b_{2}^{2}+2 b_{1} b_{2}+1+\frac{\pi^{2}}{12}$,

$A_{2}=3 a_{1} a_{3}+3 a_{2} a_{3}+b_{1} b_{3}+b_{2} b_{3}$,

$A_{3}=a_{3}^{2}+\frac{b_{3}^{2}}{3}+\frac{\pi^{2} \omega_{\mathrm{f}}^{\prime 2}}{12}$,

and

$$
\begin{aligned}
& \left.a_{1}=\frac{1}{\gamma_{1}} \frac{1}{2}+\sqrt{\frac{1}{6}} \frac{\omega_{\mathrm{f}}^{\prime}}{\gamma_{1}}\right) \quad b_{1}=\frac{1}{\gamma_{1}}\left(\sqrt{\frac{3}{8}}+\frac{\omega_{\mathrm{f}}^{\prime}}{2 \gamma_{1}}\right), \\
& \left.\left.a_{2}=\frac{1}{\gamma_{2}} \frac{1}{2}-\sqrt{\frac{1}{6}} \frac{\omega_{\mathrm{f}}^{\prime}}{\gamma_{2}}\right) \quad b_{2}=\frac{1}{\gamma_{2}}-\sqrt{\frac{3}{8}}+\frac{\omega_{\mathrm{f}}^{\prime}}{2 \gamma_{2}}\right), \\
& a_{3}=\sqrt{\frac{1}{6}} \omega_{\mathrm{f}}^{\prime}\left(\frac{1}{\gamma_{2}}-\frac{1}{\gamma_{1}}\right) \quad b_{3}=-\frac{\omega_{\mathrm{f}}^{\prime}}{2}\left(\frac{1}{\gamma_{2}}+\frac{1}{\gamma_{1}}\right) .
\end{aligned}
$$

The coupling between the frequency and the amplitude of the pulse due to the sliding filters reintroduces a $z^{3}$ dependance of the phase variance. When $\omega_{\mathrm{f}}^{\prime}=0$, the terms $A_{2}$ and $A_{3}$ are reduced to zero, and $A_{1}=3 / k_{\mathrm{f}}^{2}+1+\pi^{2} / 12$, so that we recover the result derived in [8] for fixed-frequency filters. Eqs. (10)-(12) are the main result of this letter. As for timing jitter, sliding the central frequency of the in-line filters induces a penalty on the phase fluctuations. Therefore, for a given filter strength $k_{\mathrm{f}}$, there is a tradeoff between the reduction of the continuum and the growth of the phase jitter, which both occur when the sliding rate is increased. It should be noted that Eq. (10) does not apply when the continuum is strong, because the perturbation theory does not take into account interactions between the soliton and the radiative background. To investigate the suppression of continuum, we now confront the analytical result with simulations.

Statistical simulations based on the split-step Fourier method were performed to calculate the phase jitter as a function of distance in a soliton channel. Optical pulses with a 10 ps width were propagated in a link composed of spans of dispersion-shifted fiber with $D=0.25 \mathrm{ps} /(\mathrm{nm} \mathrm{km})$ and optical amplifiers with $n_{\mathrm{sp}}=1.5$ placed every $45 \mathrm{~km}$ along the link. Gaussian filters were placed after each amplifier. The phase was numerically evaluated using the formula given in [14]

$\left.\phi=\tan ^{-1} \frac{\int|u|^{2} \operatorname{Im}(u) \mathrm{d} t}{\int|u|^{2} \operatorname{Re}(u) \mathrm{d} t}\right)$.

Fig. 1 is a plot of the theoretical and numerically obtained phase standard deviation as a function of distance in two systems with no sliding and filter bandwidths of $140 \mathrm{GHz}\left(k_{\mathrm{f}}=0.16\right)$ and $100 \mathrm{GHz}\left(k_{\mathrm{f}}=0.33\right)$. We observe that the growth of the continuum causes a large deviation of the numerical curve around $7 \mathrm{Mm}$ for the $140 \mathrm{GHz}$ system and $4.5 \mathrm{Mm}$ for the $100 \mathrm{GHz}$, and the phase jitter grows very rapidly beyond this point. As expected, the continuum grows more rapidly when the filter bandwidth is smaller because the excess gain is higher.

The standard deviation of the phase is plotted in Fig. 2 for the $140 \mathrm{GHz}$ system with sliding rates

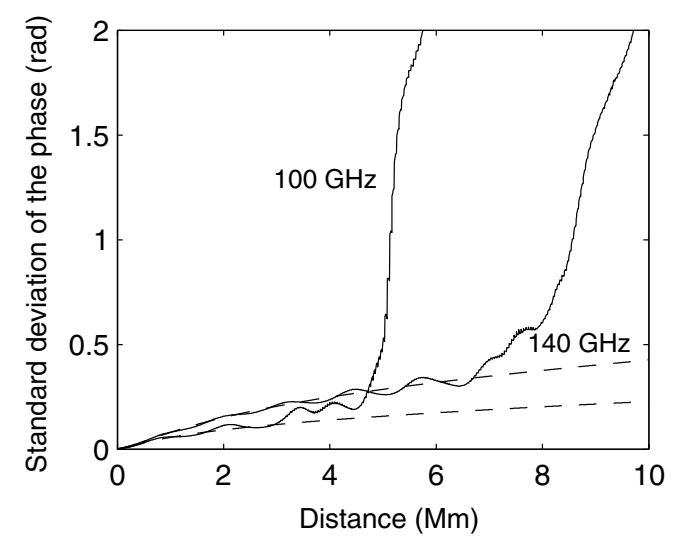

Fig. 1. Standard deviation of the phase as a function of distance for filter bandwidths of 100 and $140 \mathrm{GHz}$, and no sliding. Solid line: numerical simulation, dashed line: perturbation theory. 


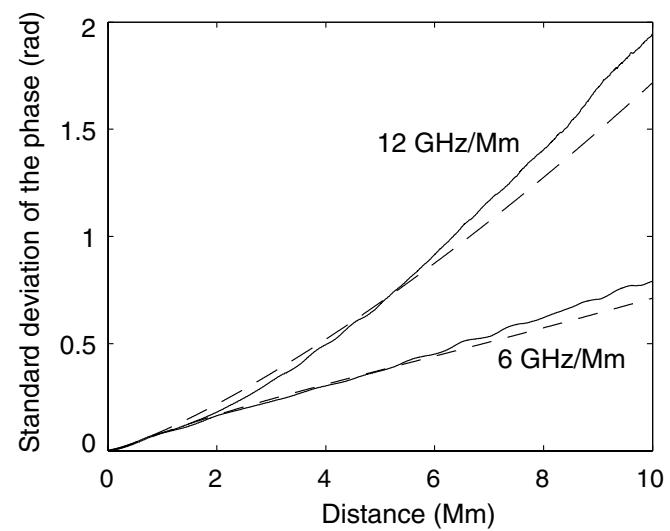

Fig. 2. Standard deviation of the phase as a function of distance for a filter bandwidth of $140 \mathrm{GHz}$ and sliding rates of 6 and $12 \mathrm{GHz} / \mathrm{Mm}$. Solid line: numerical simulation, dashed line: perturbation theory.

of $6 \mathrm{GHz} / \mathrm{Mm}\left(\omega_{\mathrm{f}}^{\prime}=0.022, \Delta=-0.13\right)$ and 12 $\mathrm{GHz} / \mathrm{Mm}\left(\omega_{\mathrm{f}}^{\prime}=0.043, \Delta=-0.26\right)$. In both systems, the sliding filters efficiently suppress the continuum, and the perturbation theory succeeds in predicting the phase jitter. For the $6 \mathrm{GHz} / \mathrm{Mm}$ sliding rate, the term in $z^{2}$ in Eq. (10) is predominant for distances in the $\mathrm{Mm}$ range, so that the phase standard deviation appears to grow linearly. When the sliding rate is further increased to 12 $\mathrm{GHz} / \mathrm{Mm}$, the term in $z^{3}$ becomes predominant for the same range of distances, so that the phase jitter is considerably increased by the sliding filters. The $100 \mathrm{GHz}$ system requires a higher sliding rate to suppress the radiative background exponential growth. Fig. 3 is a plot of the phase jitter as a function of distance in this system, with a sliding rate of $9 \mathrm{GHz} / \mathrm{Mm}\left(\omega_{\mathrm{f}}^{\prime}=0.032, \Delta=-0.10\right)$. This sliding rate allows sufficient suppression of the radiative background noise over $10 \mathrm{Mm}$. This confirms the fact that narrower filters can be used to further suppress phase jitter.

Other methods are known to mitigate the growth of nonlinear phase jitter. In-line phase conjugation [15] was shown to reduce the phase jitter. Post-transmission nonlinear phase-shift compensation [16-18] takes advantage of the correlation between amplitude and phase jitter to decrease the latter. These two methods can be used in conjunction efficiently if a symmetric phase conjugation scheme is used. Fixed or sliding in-line

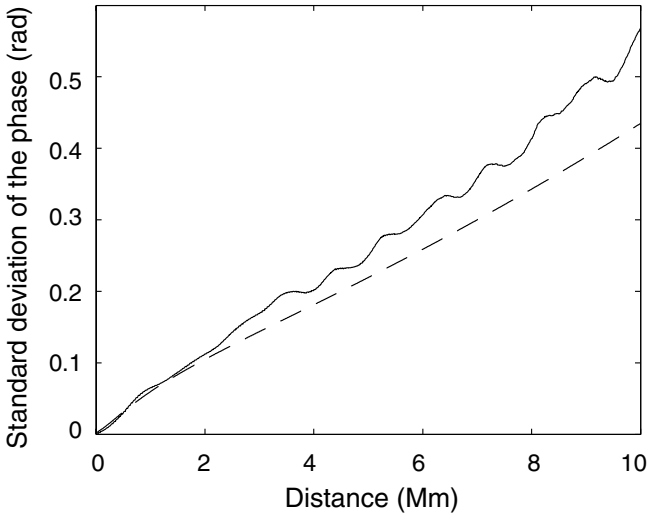

Fig. 3. Standard deviation of the phase as a function of distance for a filter bandwidth of $100 \mathrm{GHz}$ and a sliding rate of 9 $\mathrm{GHz} / \mathrm{Mm}$. Solid line: numerical simulation, dashed line: perturbation theory.

filtering has the advantage of being a purely passive process, while the other two aforementionned methods require sophisticated active nonlinear optics, such as parametric amplifiers, periodicallypoled lithium niobate integrated waveguides, or high-speed detection and phase modulation. However, nothing prevents the optical link designer from using these three methods together to obtain an optimal phase jitter reduction.

In conclusion, we have examined the effect of sliding filters on the phase jitter of solitons propagating in amplified constant-dispersion communications systems. We have shown that sliding filters reintroduce a phase jitter that grows as $z^{3}$, making them less efficient in PSK systems than they are to suppress timing jitter in OOK systems.

\section{References}

[1] A.H. Gnauck, G. Raybon, S. Chandrasekhar, J. Leuthold, C. Doerr, L. Stulz, E. Burrows, Photon. Technol. Lett. 15 (2003) 467.

[2] P.S. Cho, V.S. Grigoryan, Y.A. Godin, A. Salamon, Y. Achiam, Photon. Technol. Lett. 15 (2003) 473.

[3] K. Ishida, T. Kobayashi, J. Abe, K. Kinjo, S. Kuroda, T. Mizuochi, Optical Fiber Conference 2003, paper ThE2.

[4] J.-X. Cai, D.G. Foursa, C.R. Davidson, Y. Cai, G. Domagala, H. Li, L. Liu, W.W. Patterson, A.N. Pilipetskii, M. Nissov, N.S. Bergano, Optical Fiber Conference 2003, paper PD22.

[5] C. Xu, X. Liu, L.F. Mollenauer, X. Wei, Photon. Technol. Lett. 15 (2003) 617. 
[6] E. Iannone, F. Matera, A. Mecozzi, M. Settembre, Nonlinear Optical Communication Networks, Wiley-interscience, 1998, p. 155.

[7] C.J. McKinstrie, C. Xie, C. Xu, Opt. Lett. 28 (2003) 604.

[8] M. Hanna, H. Porte, W.T. Rhodes, J.-P. Goedgebuer, Opt. Lett. 24 (1999) 732.

[9] L.F. Mollenauer, J.P. Gordon, S.G. Evangelides, Opt. Lett. 17 (1992) 1575.

[10] F. Favre, D. LeGuen, Electron. Lett. 31 (1995) 991.

[11] L.F. Mollenauer, E. Lichtman, M.J. Neubelt, G.T. Harvey, Electron. Lett. 29 (1993) 910.
[12] L.F. Mollenauer, P.V. Mamyshev, M.J. Neubelt, Opt. Lett. 19 (1994) 704.

[13] T. Georges, Opt. Fiber Technol. 1 (1995) 97.

[14] K.J. Blow, N.J. Doran, S.J.D. Phoenix, Opt. Commun. 88 (1992) 137.

[15] C.J. McKinstrie, S. Radic, C. Xie, Opt. Lett. 28 (2003) 1519.

[16] X. Liu, X. Wei, R.E. Slusher, C.J. McKinstrie, Opt. Lett. 27 (2002) 1616.

[17] C. Xu, X. Liu, Opt. Lett. 27 (2002) 1619.

[18] K.-P. Ho, Opt. Commun. 221 (2002) 419. 